\title{
THE HARWELL NUCLEAR PHYSICS CONFERENCE, 1950
}

$\mathrm{T}$ WO hundred and thirty delegates, of whom one hundred were from overseas, attended the International Nuclear Physics Conference held in the Clarendon Laboratory and Geography School lecture theatres in Oxford during September 7-13. In the five days of meetings nearly one hundred papers were given and there was much stimulating discussion both in and outside the sessions. In the confines of a short article it is possible to mention but $a$ fraction of the papers and then only very briefly.

The sessions were divided into four main groups. On September 7 and 8, papers were given on the construction of high-energy accelerating machines and experimental work done with them, together with experiments in the allied field of cosmic radiation. The papers on September 11 and 12 were concerned with experiments at lower energies involving more modest accelerating equipment. September 13 was given to 'pile' physics, and, during the course of the meetings, alternative sessions on techniques, $\alpha$ - and $\beta$-ray spectroscopy, neutron spectroscopy and theoretical physics were held.

Sir John Cockeroft, director of the Atomic Energy Research Establishment, Harwell, after welcoming the delegates, took the chair for the opening session on high-energy accelerators. The first paper, by Prof. L. W. Alvarez, reviewed the machine programme at Berkeley, California, and gave some experimental results. In particular, the differential $p-p$ scattering cross-section has been found to have the value of $4 \mathrm{mB}$. per steradian at $90^{\circ}$ and $340 \mathrm{MeV}$., and is independent of angle and energy in the range $200-350 \mathrm{MeV}$. The 60 -in. cyclotron has been modified by Hamilton and Miller to accelerate carbon-12 nuclei and has been used by Seaborg to produce californium-244 and -246 from uranium-238. The method will probably lead to the production of further transuranium elements.

Prof. P. G. Kruger described the Illinois betatron, which was brought into operation in February and is giving an output of $1 \mathrm{R}$. per pulse, the maximum energy being $345 \mathrm{MeV}$. The Brookhaven proton synchrotron ('Cosmotron') is 80-90 per cent complete, and Prof. G. B. Collins discussed the difficulties which have to be overcome to obtain a beam.

Among machines brought into operation recently, Prof. E. T. Booth discussed the 385-MeV. Columbia cyclotron, Dr. U. Liddel (Office of Naval Research) the 340-MeV. synchrotron at the Massachusetts Institute of Technology, and Mr. M. Snowden the 170-MeV. Harwell cyclotron. At the Massachusetts Institute of Technology, experiments are planned to utilize the optical energy radiated from the accelerating electrons for studies in the region of 50-200 A., which has been neglected hitherto for lack of a suitable light source.

Meson physics was introduced by Prof. B. J. Moyer (California), who discussed the Berkeley evidence for the $\pi^{\circ}$ particle. An upper limit of $2 \times 10^{-13}$ sec. can be set for the half-life of the particle and, from experiments on the $\gamma$-rays resulting from $\pi^{-}$meson capture in hydrogen, the mass of the $\pi^{0}$ meson is found to be $261 \pm 3 m_{e}$. Capture of $\pi^{-}$mesons in deuterium does not lead to $\pi^{0}$ formation but yields neutrons according to the equations

$$
\begin{aligned}
\pi^{-}+\mathrm{D} & \rightarrow n+n, \\
& \rightarrow n+n+\gamma .
\end{aligned}
$$

Reaction (1) is twice as likely as (2).

The Bristol value for the half-life of the $\pi^{\circ}$ meson was given by Mr. D. T. King as $3 \times 10^{-14}$ sec., and Prof. G. E. Cosyns (Brussels) showed that in stars having more than twenty penetrating particles there is angular correlation between pairs. His results suggest, he said, that both multiple and plural production of mesons occur. Evidence for the $V$-mesons (both charged and uncharged) obtained by the Manchester group and by Prof. Anderson's group (California) was presented by Prof. P. M. S. Blackett, who suggested tentative decay schemes.

Further scattering and high-energy disintegration experiments were reported from Bristol, Harvard, Harwell and Rochester, but these will not be discussed here.

The theoretical session on high-energy physics was enlivened by the challenge of the new $p-p$ and $n-p$ scattering data where the results are in disagreement with the theory. Dr. A. Pais (Princeton) gave an admirable review of the position, and showed that the experiments can only be explained by introducing a strongly singular force between protons and a somewhat smaller one between neutron and proton. $\mathrm{He}$ stated that for spin-orbit coupling he favoured the potential well

$$
\varphi(r) \propto \frac{1}{r} \frac{d}{d r}\left(\frac{\exp -K r}{r}\right)
$$

which is the meson-theoretical analogue of the potential encountered in atomic theory.

After a visit to the laboratories of the Atomic Energy Research Establishment, Harwell, on September 9, the week-end was taken up with social activities.

The Conference resumed on September 11, when Prof. T. Lauritsen, after describing the machines and spectrometers in use at the California Institute of Technology, gave up-to-date level schemes for oxygen-16, lithium-7 and the interesting mirror nucleus beryllium-7, the $\gamma$-ray from the $430-\mathrm{keV}$. level of which has now been observed. Reactions resulting from the bombardment of fluorine by protons have received much attention in the past, but new work is still being reported. Prof. S. Devons (London) gave results on the angular correlation between the $\alpha$-particles and the pairs from the pairemitting state of oxygen-16 which agree with Dirac theory assuming a spherically symmetrical field, and Mr. J. G. Rutherglen (Glasgow) showed evidence for a 12 - $\mathrm{MeV}$. $\gamma$-ray which suggests a low-lying level in the neon nucleus at $1.53 \pm 0.1 \mathrm{MeV}$.

An ionization chamber in which wall effects have been eliminated has been used in Heidelberg for the study of energy-levels excited by a fast neutron flux. It was described by Prof. W. Bothe, who reported finding many new excited states of nitrogen-15. The reactions $\mathbf{O}^{16}(d p) \mathrm{O}^{17}, \mathrm{~F}^{10}(d \alpha) \mathrm{O}^{12}$ and $\mathbf{C}^{12}(d p) \mathbf{C}^{13}$ have been used by Prof. J. Rotblat (London) to obtain level-schemes for carbon-13 and oxygen-17. 
The results show a remarkable correlation between the energy states of the two nuclei for which it is difficult to account on theoretical grounds.

The major part of the afternoon session was devoted to the study of reactions involving tritium, either as a target or a bombarding particle. Prof. T. Bonner (Rice Institute), Dr. R. F. Taschek (Los Alamos Scientific Laboratory) and Mr. M. J. Poole (Harwell) have all investigated the energy-dependence of the cross-section for the $\mathrm{D}(\mathrm{T} n) \mathrm{He}^{4}$ reaction. It has a maximum value of about 5 barns in the region of $180-\mathrm{keV}$. triton energy. Mr. B. H. Flowers (Harwell) showed that strong spin-orbit coupling is necessary to account for such a large cross-section, and the calculated energy-dependence agrees with the experimental results if the accurate wave-functions of the Coulomb field are employed. Los Alamos experiments on the reactions $\mathrm{T}(p n) \mathrm{He}^{3}$ and $\mathbf{T}(p \gamma) \mathrm{He}^{4}$ suggest the existence of a helium-4 level above $20 \mathrm{MeV}$. The T-T reaction, among the possible products of which are the dineutron and helium-5, is being studied at Los Alamos and Chalk River, but the experiments do not allow definite conclusions to be drawn at present.

A paper on $(p \gamma)$ coincidence experiments using scintillation detectors having high resolution was given by Dr. W. E. Burcham (Cambridge). Applied to the reaction $\mathrm{B}^{10}(d p) \mathrm{B}^{11}$, counting-rates as high as 10 coincidences per microcoulomb of deuterons have been obtained.

The morning session of September 12 on angular distribution experiments and their interpretation was introduced by Prof. D. R. Inglis (Argonne National Laboratory), who outlined the theory with particular reference to the reactions $\mathrm{Li}^{7}(p \alpha) \mathrm{He}^{4}$ and $\mathrm{Li}^{6}(d \alpha) \mathrm{He}^{4}$. Analysis of these reactions shows that parities deduced from the nuclear models are correct; but disappointment was expressed that most of the information obtained relates to the highly excited state of the compound nucleus, which is in a region too complex to be treated theoretically. In a review of $p-p$ and $n-p$ scattering experiments in the region up to $30 \mathrm{MeV}$., Prof. J. H. Williams (Minnesota) showed they can be interpreted assuming that the purely nuclear scattering occurs only in states of angular momentum zero. Recent Minnesota experiments demonstrate that, in the region below $5 \mathrm{MeV}$., the 'effective range' between nucleons is independent of charge.

Dr. H. Wäffler (Zurich) described measurements of the separate angular distribution of the lithium 17.6and $14 \cdot 8-\mathrm{MeV} . \gamma$-rays, from which he concluded that the $17 \cdot 6-\mathrm{MeV}$. level of beryllium 8 has even parity and odd angular momentum. Prof. P. Huber (Basel) discussed an experiment on the differential scattering cross-section of neutrons of about $3 \mathrm{MeV}$. energy by carbon, nitrogen and oxygen nuclei.

Related papers on the angular distribution of neutrons and protons from $(d n)$ and $(d p)$ reactions were given by Dr. J. Holt (Liverpool), who showed. that there is a strong forward peak in both types of reaction, and by Prof. Rotblat, who has examined the angular distribution of the protons in $(d p)$ reactions for various states of the final nucleus. The theory of these processes was treated by Mr. S. T. Butler (Birmingham), who showed how the angular distributions can be used to give the spins and parities of the nuclei involved. He invokes a stripping process and, by inserting certain boundary conditions, can predict the angular distributions to be expected for various values of spin and parity of the state of the final nucleus when these properties are known for the ground-state of the target nucleus. As an example he found from the experiments that the ground-state of oxygen-17 should be a $D_{3 / 2}$ or $D_{5 / 2}$ state.

The afternoon session on photodisintegration experiments was largely devoted to experiments with the deuteron. Dr. H. von Halban reviewed recent work in Oxford which still shows discrepancies with theory, and Dr. D. H. Wilkinson described Cambridge experiments with $\gamma$-rays extending from 4. to $17.6 \mathrm{MeV}$. and which lead to a value of the triplet range in good agreement with the figure reported later by Dr. Hughes. The disintegration $\mathrm{C}^{12}(\gamma 3 \alpha)$ was described by Dr. V. Telegdi (Zurich), who found that, after going through a maximum at 18.5 MeV., the cross-section rises again as the energy is increased to $32 \mathrm{MeV}$. Mr. F. K. Goward (Harwell) discussed work on the reaction $\mathrm{O}^{16}(\gamma 4 \alpha)$, which proceeds either as a $(\gamma \alpha)$ reaction via an excited carbon-12 nucleus or as a fission-like process in which the oxygen nucleus breaks into two beryllium-8 nuclei. A new reaction, $\mathrm{Li}^{7}(\gamma \mathrm{T}) \mathrm{He}^{4}$, was reported by $\mathrm{Dr}$. E. W. Titterton (Harwell), who gave a value for the cross-section of $1.2 \pm 0.6 \times 10^{-28} \mathrm{~cm}^{2}$ at $17.6 \mathrm{MeV}$., and showed an excitation function indicating that the cross-section has a maximum value in the neighbourhood of $14 \mathrm{MeV}$.

The 'pile' physics sessions on September 13 were more concerned with research done with neutron beams than with the physics of piles. Dr. R. E. Bell described the Chalk River heavy-water pile, the neutrons from which have been used to excite $\gamma$-rays from neutron capture in hydrogen. From a measurement of their energy, the binding energy of the deuteron was found to be $2 \cdot 230 \pm 0 \cdot 007 \mathrm{MeV}$., which is significantly higher than earlier values. The elegant experiments of J. M. Robson demonstrating the $\beta$-decay of the neutron were given detailed treatment. An allowed spectrum is found, the end-point being at $782 \mathrm{keV}$. Dr. D. J. Hughes described the Argonne $300-\mathrm{kW}$. pile and the newly completed Brookhaven 30-MW. pile. A high-speed mechanical chopper will be used with the latter for neutron time-of-flight experiments over a path-length of 20 metres. The device is expected to give a resolution ten times better than that of any previous chopper. Slowneutron scattering experiments have allowed determination of the nuclear interaction triplet range as $1.74 \pm 0.1 \times 10^{-13} \mathrm{~cm}$. and the singlet range as $2 \cdot 6 \pm 0 \cdot 5 \times 10^{-13} \mathrm{~cm}$.

The Oak Ridge pile has been used for experiments on neutron diffraction and the decay of the neutron. Dr. A. H. Snell reported on both and gave scattering cross-sections and scattering efficiencies for various nuclei as measured by Wollan and Shull. At Los Alamos, the water boiler and fast reactor both operate at $10 \mathrm{~kW}$. and give $10^{11}$ (slow) and $5 \times 10^{12}$ (fast) neutrons $/ \mathrm{cm} .^{2} / \mathrm{sec}$. respectively. After describing them, Dr. D. K. Froman discussed a proposed experiment to investigate the angular distribution of neutrons scattered by liquid helium II which, it is expected, will determine the statistics of the scattering nuclei.

Pile modulation techniques for cross-section measurement were discussed by Mr. D. J. Littler (Harwell) and Dr. L. Kowarski (Paris); and a stimulating paper on neutron beam magnetic resonance experiments was given by Prof. N. F. Ramsey (Harvard), who is currently using the method to determine the electric dipole moment of the neutron and the 
neutron magnetic moment. Fission physics were treated in three papers: the first by Dr. J. C. Jacobsen (Copenhagen) on the fluctuations of charge on the fission fragments, the second on rare modes of fission by Dr. E! W. Titterton (Harwell) and the last, by Mr. J. Scott (Cambridge), on the theory of the fission process.

$\beta$-ray spectroscopy was treated in an alternative session. Dr. K. Siegbahn outlined the work in Stockholm on the internal conversion coefficients of certain isomeric transitions; he finds the results to be compatible with those expected on the shell model. Dr. H. O. W. Richardson described the spectrometers in use in Edinburgh, and Dr. S. C. Curran gave details of experiments in Glasgow, using proportional counters placed in a magnetic field, to examine $\beta$ - and $\gamma$-ray spectra up to an energy limit of $2 \mathrm{MeV}$. Work at Harwell on gold-198 was reported by Dr. P. E. Cavanagh, and an experiment determining the internal conversion coefficient of an isomeric transition in bromine-82 was described by Mr. D. West. In the field of $\alpha$-ray spectroscopy, experiments reported by Dr. S. Rosenblum (Paris) provided information on the nuclear radii of the $4 n, 4 n+1$ and $4 n+3$ radioactive series.

The neutron spectroscopy session included discussion of three types of time-of-flight spectrometer : the direct method of the mechanical modulator ('chopper') was described by Dr. Hughes; the modulated cyclotron, typified by the Columbia installation, was discussed by Prof. W. W. Havens, who also reported a new measurement of the electronneutron interaction potential as $5,000 \pm 1,000 \mathrm{eV}$.; and Mr. A. W. Merrison discussed the Harwell equipment which employs a pulsed linear electron accelerator as a photo-neutron source. In addition, papers were given on neutron diffraction by Prof. $K$. Lonsdale (London), Dr. D. G. Hurst (Chalk River) and Mr. G. E. Bacon (Harwell).

The theoretical physics session was devoted to discussion of possible models of nuclear structure and their mutual relation. Prof. R. E. Peierls (Birmingham) explained some of the difficulties confronting the 'compound nucleus' picture, formulated first by Bohr ; Prof. E. Wigner (Princeton) outlined the evidence which produced the concept of 'magic numbers', and the shell-structure models of the nucleus which have been suggested to explain these numbers ; Prof. Inglis reported further work on the $j-j$ coupling model. Prof. Peierls also directed attention to recent significant experiments at Chalk River by Kinsey et al., which showed that some heavy nuclei have entirely unexpected properties more characteristic of simple light nuclei. There was considerable discussion on the relation of the shellstructure model to other models, and on the extent to which it might be explained by current ideas on nuclear forces, or whether now ideas had to be introduced to explain observed regularities. Prof. P. A. M. Dirac (Cambridge), outlining his new ideas in field theory, was of the opinion that a new fundamental concept is needed, and suggested it is more likely to be forthcoming as a result of studying particles about which we know most, such as photons and electrons, rather than the more obscure mesons.

The Conference dinner, presided over by Lord Cherwell, was held in the Great Hall of Christ Church on September 12, and was notable for fine afterdinner speeches by Sir John Cockeroft, Prof. S. K. Allison (Chicago) and Prof. Niels Bohr (Copenhagen).

$$
\text { E. W. Trtwerton }
$$

\section{THE CONCEPT OF CULTURE}

$\mathrm{V}$

ERY appropriately, the proceedings of Section $\mathrm{H}$ (Anthropology) of the British Association began on August 31 at Birmingham with a discussion meeting on the concept of culture. This concept has recently acquired a somewhat indiscriminate currency among anthropologists, archæologists, psychologists and other social scientists. It has dominated the development of anthropology ever since Tylor, with a stroke of genius, borrowed the term 'culture' from German scholars and generalized it to include "that complex whole which includes knowledge, belief, art, morals, law, custom, and any other capabilities and habits acquired by man as a member of society"1. It has also remained the main link between the different branches of anthropology and allied disciplines as these have become more specialized in methods and subject-matter.

The meeting was opened by Dr. Phyllis Kaberry (University College, London) with a paper on the recent history of the concept in British anthropology. She pointed out that since Tylor's day social anthropology has been defined as the science of culture, a term covering the whole way of life of an aggregate of people inhabiting a particular locality. Artefacts, ideas, institutions, and modes of organisation in social and political groupings are all included in culture, which, as Tylor implied, constitutes a system of interrelated parts. This idea lay behind the crucial change which occurred at the beginning of this century when anthropologists turned from tracing hypothetical origins and evolutionary stages to intensive field studies of existing cultures viewed as complex functioning wholes. Malinowski and Radcliffe-Brown had exceptional influence on this development in Great Britain ; and it is significant that up to about 1931 they appeared to have a common approach. Both described social anthropology as the study of culture, their major contribution being their emphasis on culture as "an integrated system" ${ }^{2}$ and their insistence that customs, institutions, etc., must be explained, not by conjectural history, but by the part they play "within an integral system"'s. The principles and concepts put forward by them has determined the type of fieldwork done by British anthropologists since 1920. The monographs of professional social anthropologists in the intervening years are not only rich in detail but also provide analyses of the nature and function of particular institutions in selected societies within the framework laid down by Malinowski and Radcliffe-Brown. Even recent work avowedly concerned with clarifying the concept of social structure must, from this point of view, be regarded as an important contribution to our understanding of culture.

But this work, continued Dr. Kaberry, is also a sign of a shift which gives cause for apprehension. A first break with the accepted view came when Malinowski himself, in 1931, outlined the theory later elaborated in his posthumous book, "A Scientifio Theory of Culture"4. He now defined culture as "an instrumental reality which has come into existence to satisfy the [biological] needs of man", and for his former sociological definition of "function" substituted one in terms of the satisfaction of primary or derived needs. Obviously, biological, psychological and environmental factors enter as determinants in the universal occurrence of many social institutions, but in Dr. Kaberry's opinion social anthropology 